\title{
OCT imaging of choroidal neovascularisation and its role in the determination of patients' eligibility for surgery
}

\author{
A Giovannini, G P Amato, C Mariotti, B Scassellati-Sforzolini
}

\begin{abstract}
Aim-To evaluate the optical coherence tomographic characteristics of choroidal neovascularisation (CNV) in age related macular degeneration (AMD) and in idiopathic and inflammatory $\mathrm{CNV}$. The use of this technique in the selection of patients for surgery is discussed.

Methods-Ocular coherence tomography (OCT), fluorescein, and indocyanine green angiography were performed in 23 patients affected by AMD complicated by well defined CNV and in 10 patients affected by inflammatory or idiopathic CNV. The neovascular membrane was surgically removed in five age related CNVs, two inflammatory choroidopathies, and two idiopathic CNVs.

Results-In inflammatory and idiopathic CNV, the OCT displayed a neovascularisation on the retinal pigment epithelium (RPE). In three cases the CNV was excised with an improvement of visual acuity equal to or greater than two Snellen lines; in a fourth case, the visual acuity after surgery was unchanged. In the cases of AMD the OCT fell into three different patterns: (A) CNV above the RPE (five cases); (B) focal, irregular thickening of the retinal pigment epithelial band (12 cases); (C) CNV above and below the RPE (six cases). The five pattern A CNV patients underwent the surgical excision of the neovascularisation. In four cases the visual acuity improved by two or more Snellen lines; in the fifth case the visual acuity remained unchanged.
\end{abstract}

Conclusions-The authors suggest that the surgical removal of early age related CNV could be performed in those cases where the OCT shows a neovascular membrane on the RPE, as in idiopathic and inflammatory CNVs.

(Br F Ophthalmol 1999;83:438-442)

Ophthalmology

University of Ancona,

Italy

A Giovannini

G P Amato

C Mariotti

B Scassellati-Sforzolini

Correspondence to:

A Giovannini, Clinica

Oculistica dell'Università di

Ancona, Nuovo Ospedale

Regionale di Torrette, 60020

Ancona, Italy.

Accepted for publication 10 November 1998 the CNV in the young, appear to be related to the integrity of the adjacent RPE and to the area of growth over the $\mathrm{RPE}^{1-5}$ On the contrary, the unsatisfactory visual results observed in the course of age related macular degeneration (AMD) seem to be related to the widespread alteration of the RPE, conditioning a significant loss of RPE cells and choriocapillaris, and with the differing relation between CNV and RPE..$^{1-4}$ 6-12

Optical coherence tomography (OCT) is a new imaging tool providing a non-invasive, non-contact in vivo cross sectional imaging of eye tissues with a maximal longitudinal resolution of $10 \mu \mathrm{m} .{ }^{13-18}$ The purpose of this study was to evaluate the optical coherence tomographic characteristics of CNV and the possible use of this technique in the selection of patients eligible for surgery.

\section{Methods}

Twenty three cases of well defined CNV complicating AMD (11 males and 12 females; aged 51-89 years; mean age 71.3 ) and 10 cases of inflammatory or idiopathic CNV (two males and eight females; aged 19-49 years; mean age 32.2) were studied. In nine cases (five age related CNVs, two inflammatory choroidopathies, and two idiopathic CNVs) the surgical excision of the neovascular membrane was performed. The selection criteria for surgery in the AMD group were: subfoveal CNV, juxtafoveal CNV with blood masking the foveal edge, juxtafoveal CNV where the centre of the fovea risked being damaged by laser photocoagulation. All CNVs were small (smaller than one optic disc diameter). Another criterion was the OCT imaging of the CNV: only the CNVs that could be identified above the layer of the RPE were eligible for surgery. The selection criteria for surgery in the case of inflammatory or idiopathic $\mathrm{CNV}$ were: subfoveal $\mathrm{CNV}$, juxtafoveal $\mathrm{CNV}$ with blood masking the foveal edge, juxtafoveal $\mathrm{CNV}$ where the centre of the fovea risked being damaged by laser photocoagulation.

All patients underwent OCT (Humphrey Instruments, Zeiss), fluorescein, and indocyanine green (ICG) angiography (Topcon IMAGEnet H1024). In those patients where the $\mathrm{CNV}$ was removed, the examinations were repeated within 3 months after surgery.

OCT was always performed by the same examiner through a dilated pupil. A superluminescent diode light operating at $830 \mathrm{~nm}$, coupled to a fibre optic delivery system, provides the necessary illumination on the retina (approximately $200 \mu \mathrm{W}$ ). The time of delay of light reflected from different depths in tissue is determined using interferometry. A false colour tomogram of optical reflectivity is 

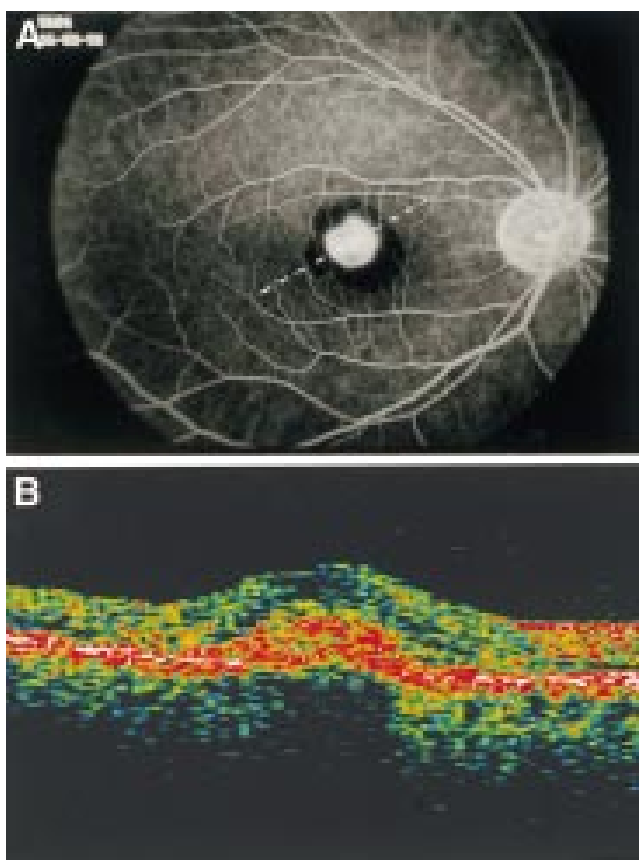

Figure 1 (A) Fluorescein angiogram shows an idiopathic subfoveal CNV in a 34 year old woman. (The broken line on FA frame indicates the OCT scan direction). (B) The OCT shows a marked thickening $(481 \mu \mathrm{m})$ of the neurosensory retina corresponding to the CNV. The CNV can be identified as an area of hyperreflectivity lying above the band corresponding to the RPE.

produced to enhance the differentiation of retinal layers. Bright colours (for example, red and white) correspond to highly reflective areas and dark colours (for example, blue and black) represent regions with low reflectivity.

All scans were separately reviewed and analysed by two observers (AG and GPA).

\section{Results}

In all the cases of inflammatory or idiopathic CNV the OCT displayed, at the same site of the CNV identified by fluorescein angiography, a focal non-homogeneous area of hyperreflectivity lying above the OCT band corresponding to the RPE. The neovascular complex showed an infiltrate-like appearance on the OCT scans of the neuroretina (Fig 1). The anterior profile of the neuroretina usually appeared elevated and sometimes with a convex shape. At the level of the CNV the neuroretinal thickness constantly increased (Table 1) as a result of retinal oedema, visualised on OCT as an area of non-homogeneous hypo-
Table 1 OCT measurement of the neuroretinal thickness $(\mu \mathrm{m})$ at the level of the choroidal neovascularisation $(C N V)$, before and after the surgical removal of the neovascular membrane

\begin{tabular}{lll}
\hline & $\begin{array}{l}\text { Before } \\
\text { surgery }\end{array}$ & $\begin{array}{l}\text { After } \\
\text { surgery }\end{array}$ \\
\hline Case 1: idiopathic CNV & 481 & 162 \\
Case 2: idiopathic CNV & 397 & 159 \\
Case 3: multifocal choroidopathy & 475 & 160 \\
Case 4: multifocal choroidopathy & 400 & 171 \\
Case 5: AMD & 269 & 154 \\
Case 6: AMD & 379 & 154 \\
Case 7: AMD & 503 & 168 \\
Case 8: AMD & 496 & 180 \\
Case 9: AMD & 305 & 168 \\
Median (SD) & 411.7 (79.88) & $164(8.04)$ \\
\hline
\end{tabular}

reflectivity. An optically empty space corresponding to a neurosensory detachment was often observed above or near the CNV. The reflectivity of the retinal pigment epithelial band adjacent to the CNV was usually homogeneous and did not present alterations, except when the scanning probe beam encountered an atrophic spot or a chorioretinal scar. In the case of atrophic spot (multifocal choroidopathies) the retinal pigment epithelial band appeared interrupted and a deeper hyperreflectivity could be seen, because of a greater penetration of the beam (Fig 2). In three cases the hyperreflectivity, that was interpreted as the OCT imaging of the CNV, was no longer visible after surgery (Fig 3 ). In the fourth case, where fluorescein angiography revealed a persistent CNV, the OCT scan (performed in "repeat mode" through the residual hyperfluorescence) displayed an abnormal hyperreflectivity above the band corresponding to the RPE choriocapillaris. The area of hyperreflectivity was smaller than that visible before surgery. This area could be interpreted as the OCT image of the persistent CNV, not completely removed by surgery. In the first three cases an improvement of visual acuity equal to or greater than two Snellen lines was observed. In the fourth case the visual acuity remained unchanged.

As regards the cases of AMD the interpretation of the OCT imaging of the CNV was more challenging. Three different OCT patterns were observed.

In five cases a focal area of hyperreflectivity could be very clearly identified above the RPE, with characteristics similar to those of inflammatory or idiopathic CNV (Fig 4). In all the cases the size of the CNV, evaluated in the early and mid phases of fluorescein angiography, was
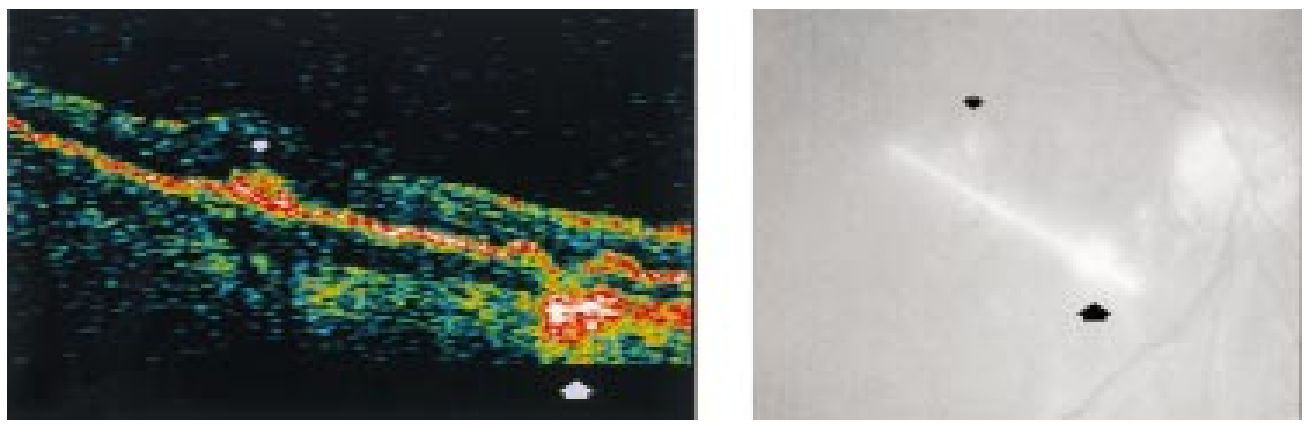

Figure 2 Multifocal choroidopathy. The OCT displays a focal hyperreflectivity lying above the RPE corresponding to the CNV (small arrows); at the same site of the atrophic spot a deep hyperreflectivity can be detected (large arrows). 

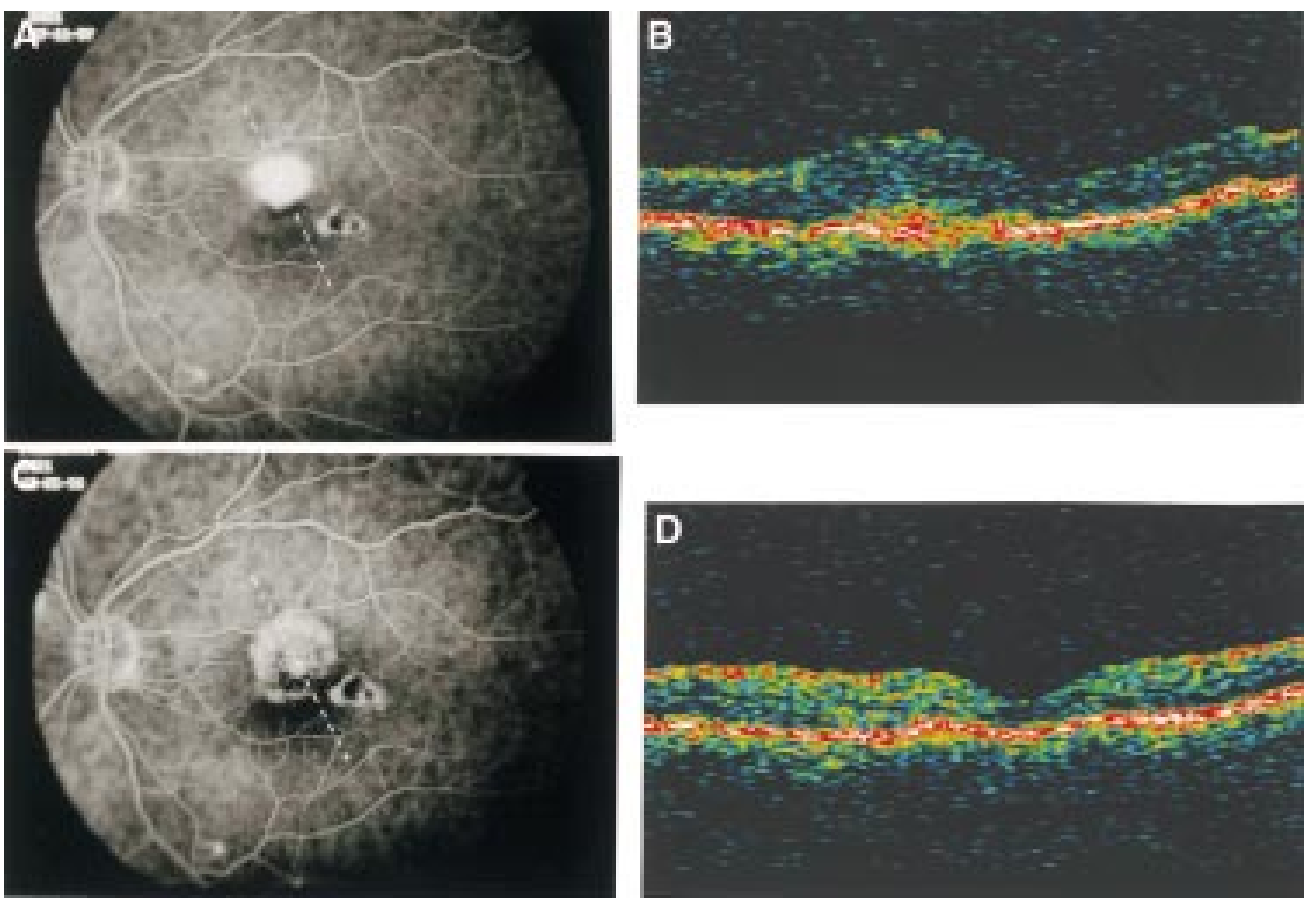

Figure 3 Multifocal choroidopathy. (A) Fluorescein angiography before surgery $(V A=20 / 40)$. (The broken line on $F A$ frame indicates the OCT scan direction). (B) OCT before surgery: the neurosensory thickness is $475 \mu \mathrm{m}$; a focal hyperreflectivity lying above the band corresponding to the RPE can be visualised. (C) Fluorescein angiography after surgery $(V A=20 / 30)$ : the CNV has been completely removed. (The broken line on FA frame indicates the OCT scan direction). (D) OCT after surgery: the normal foveal profile is restored and retinal thickness is $160 \mu \mathrm{m}$. The hyperreflectivity interpreted as the $C N V$ is no longer visible.

inferior to one disc diameter and the symptoms had been present for less than 2 months before our observation.

In 12 cases the OCT showed only a focal, irregular thickening of the band corresponding
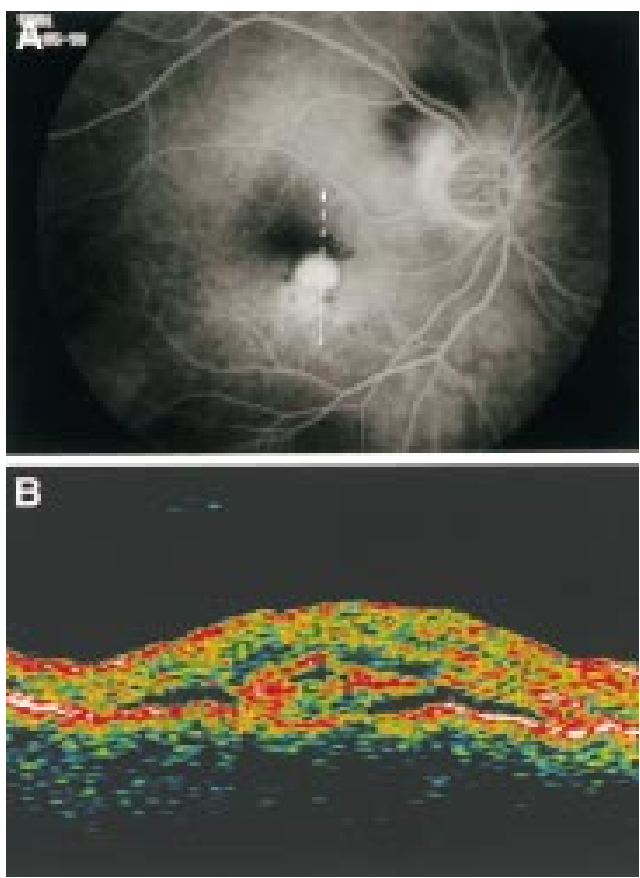

Figure 4 AMD complicated by CNV (OCT pattern A). (A) Fluorescein angiography shows a juxtafoveal and a peripapillary CNV. (The broken line on FA frame indicates the OCT scan direction). (B) The OCT scan performed through the juxtafoveal $C N V$ reveals a marked thickening $(503 \mu \mathrm{m})$ of the neurosensory retina and an increased reflectivity above the RPE interpreted as the CNV. A thin optically empty space, signifying neurosensory detachment, surrounds the CNV. to the RPE that appeared fragmented and disorganised (Fig 5).

In six cases the focal, irregular hyperreflectivity could be identified both above and below the level of the band corresponding to the RPE. Sometimes the RPE band seemed to present a focal duplication (Fig 6).

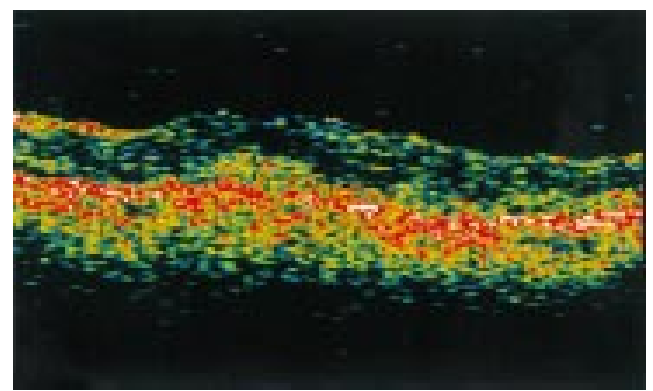

Figure 5 AMD complicated by CNV (OCT pattern B). The OCT shows a focal, irregular thickening of the band corresponding to the RPE, that appears fragmented and disorganised.

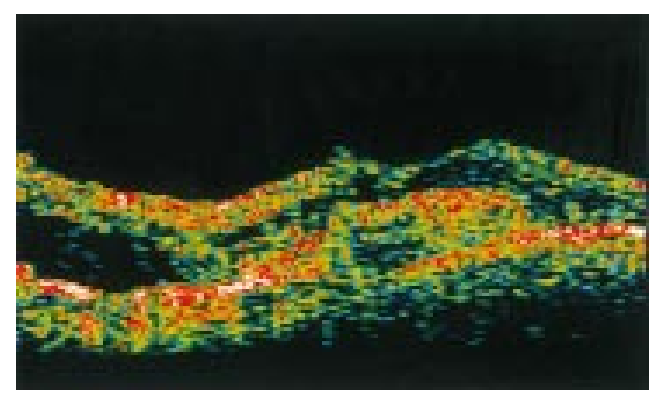

Figure 6 AMD complicated by CNV (OCT pattern C). An irregular hyperreflectivity can be visualised both above and below the level of the band corresponding to the RPE. The retinal pigment epithelial band presents a focal duplication. 

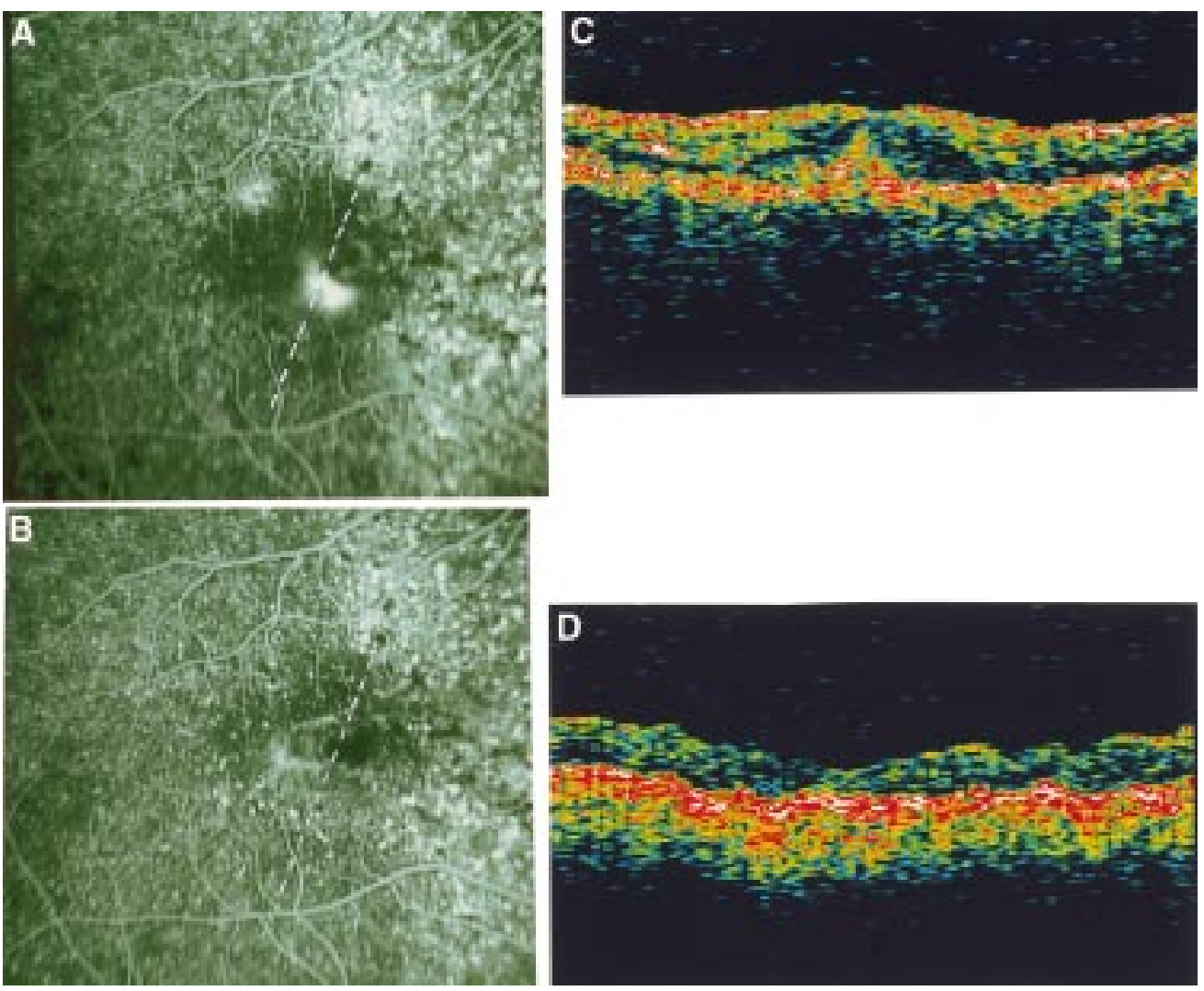

Figure 7 AMD complicated by subfoveal CNV. (A) Fluorescein angiography before surgery (VA=20/200). (The broken line on FA frame indicates the OCT scan direction). (B) The OCT clearly displays a focal hyperreflectivity lying above the $R P E$ interpreted as the CNV. In this case the OCT shows the relation between CNV and the centre of the fovea. The adjacent neuroretina presents a non-homogeneous, reduced, hyperreflectivity due to oedema. The retinal thickness is $379 \mu \mathrm{m}$. (C) Fluorescein angiography after surgery $(V A=20 / 60)$. The loss of retinal pigment epithelial cells and choriocapillaris is minimal. (The broken line on FA frame indicates the OCT scan direction). (D) The focal hyperreflectivity identified by OCT before surgery is no longer visible. The retinal thickness is $154 \mu \mathrm{m}$.

The $\mathrm{B}$ and $\mathrm{C}$ patterns were observed in the cases of CNV greater than one disc diameter, with symptoms dating from over 3 months before our observation.

The RPE adjacent to the CNV presented widespread alterations of the reflectivity and of its profile, which appeared irregular and non-linear. Drusen could often be identified.

Neurosensory detachments were constantly present in pattern $\mathrm{B}$ and $\mathrm{C}$ cases. In pattern $\mathrm{A}$ cases the detachment was smaller and not always present.

In five cases of $\mathrm{CNV}$ complicating $\mathrm{AMD}$ lying on the RPE (pattern A), the surgical removal of the neovascular membrane was performed (Fig 7). Similar to what was observed in young patients, the OCT abnormalities that were considered as CNV were no longer detectable after surgery and the normal profile of the anterior neuroretina was restored (Table 1). The normalisation of the OCT picture was followed by an improvement of visual acuity greater than two Snellen lines in four cases. In the fifth case (subfoveal CNV) the visual acuity remained unchanged.

\section{Conclusions}

Fluorescein angiography remains the essential tool in the diagnosis of CNV. In some cases of occult CNV of the elderly, ICG angiography has been shown to be useful. ${ }^{19}{ }^{20}$ The interpretation of the OCT imaging of CNVs complicat- ing AMD is very difficult. In fact the neovascular membrane, $\mathrm{RPE}$, and choriocapillaris are structures with similar reflectivities ${ }^{21}$ and the identification of the single structures is often not easy. In our experience the difficulties increase in cases of long lasting and larger sized CNVs (that is, greater than one disc diameter). In such cases the OCT is rarely useful in the identification of the neovascular complex. In agreement with Puliafito et $a l,{ }^{22}$ the RPE was irregularly thickened and disrupted and the CNV could not be recognised. Only in the cases of CNV smaller than one disc diameter and symptoms dating from less than 2 months before, an anomalous hyperreflectivity over the RPE was visualised using OCT. The OCT imaging in these cases looks quite similar to that of inflammatory and idiopathic CNV.

In all the cases of our series of inflammatory and idiopathic CNV, the growth was always above the RPE; on the contrary in the cases of AMD the relation between CNV and RPE did not present the same pattern. In five cases the CNV could be visualised above the RPE, in 12 cases the OCT displayed a focal, irregular thickening of the RPE and in six cases a focal, irregular hyperreflectivity was seen both above and below the RPE. OCT seemed to confirm the most recent anatomopathological studies of surgically excised CNVs; these studies have shown that in AMD the CNV may grow not only below the RPE but also above it or in a 
combined pattern. ${ }^{23} 24$ On the contrary, in inflammatory $\mathrm{CNV}$ the neovascularisation was found above the RPE. ${ }^{5}$

In the five cases of AMD where the CNV was visualised above the RPE, the onset of the symptoms was recent (less than 2 months before the observation) and the size was less than one optic disc diameter. On the contrary none of the remaining 18 cases with longstanding and large CNV showed the pattern of growth of the CNV above the RPE. It could be therefore hypothesised that, in the cases of $\mathrm{AMD}$, the well defined CNV may grow above the RPE at its onset, as in idiopathic and inflammatory cases. Subsequently, in the course of the disease, the growth of the neovascular membrane could lead to a widespread disorganisation of the RPE layer, owing to the characteristics of the RPE in elderly patients. A study on a larger series of patients affected by AMD complicated by a CNV smaller than one optic disc diameter would be necessary to assess, with a statistical significance, the prevalence of pattern A CNVs (those growing above the RPE) within the group of patients with small CNVs of recent onset.

The disappearance, after surgery, of the hyperreflectivity considered as CNV in three out of four cases of our series, in agreement with fluorescein angiography, would be additional proof that the images interpreted as CNVs were in fact neovascular membranes.

The aforementioned considerations could be important for the surgical removal of the CNV. In fact the benefit of the surgical excision of the CNV is related not only to the conditions of RPE and choriocapillaris, but also to the relation between RPE and CNV growth. The location of the CNV may therefore have an influence on the number of $\mathrm{RPE}$ and choriocapillaris cells damaged by the surgical approach. The good visual results after surgery of inflammatory CNVs, are due to the fact that the CNV lies above the RPE. This condition allows the surgeon to remove the membrane with minimal damage to RPE and choriocapillaris. The surgical excision of early age related CNVs could be suggested in those cases where, as in idiopathic and inflammatory CNVs, the neovascular membrane lies above the RPE. The authors suggest that OCT imaging of CNVs complicating AMD could be a useful tool in the selection of cases eligible for surgery.

1 Thomas MA, Grand MG, Williams DF, et al. Surgical management of subfoveal choroidal neovascularization. $O p h-$ agement of subfoveal choro
thalmology 1992;99:952-68.
2 Berger AS, Kaplan HJ. Clinical experience with the surgical removal of subfoveal neovascular membranes. Ophthalmolremoval of subfoveal
ogy 1992;99:969-76.

3 Gass JDM. Biomicroscopic and histopathologic considerations regarding the feasibility of surgical excision of subfoveal neovascular membranes. Am 7 Ophthalmol 1994; 118:285-98.

4 Thomas MA, Dickinson JD, Melberg NS, et al. Visual results after surgical removal of subfoveal choroidal neovascular membranes. Ophthalmology 1994;101:138496.

5 Berger AS, Conway M, Del Priore LV, et al. Submacular surgery for subfoveal choroidal neovascular membranes in patients with presumed ocular histoplasmosis. Arch Ophthalmol 1997;115:991-6.

6 Lambert HM, Capone A, Aarberg TM, et al. Surgical excision of subfoveal neovascular membranes in age-related sion of subfoveal neovascular membranes in age-related

7 Ormerod LD, Puklin JE, Frank RN. Long term outcomes after surgical removal of advanced subfoveal neovascular membranes in age-related macular degeneration. Ophthalmology 1994;101:1201-10.

8 Nasir MA, Zarbin MA. Choriocapillaris atrophy as a complication of surgical excision of choroidal neovascular membranes. Invest Ophthalmol Vis Sci 1993;34:653.

9 Grossniklaus HE, Hutchinson AK, Capone A, et al. Cilinicopathologic features of surgically excised choroidal neovascular membranes. Ophthalmology 1994;101:1099111 .

10 Hudson HL, Frambach DA, Lopez PF. Relation of the functional and structural fundus changes after submacular surgery for neovascular age-related macular degeneration. Br F Ophthalmol 1995;79:417-23.

11 Pollack JS, Del Priore LV, Smith ME, et al. Postoperative abnormalities of the choriocapillaris in exudative agerelated macular degeneration. Br f Ophthalmol 1996;30: 314-18.

12 Nasir MA, Sugino I, Zarbin MA. Decreased choriocapillaris perfusion following surgical excision of choroidal neovascular membranes in age-related macular degeneration. $\mathrm{Br} F$ Ophthalmol 1997;81:481-9.

13 Huang D, Swanson EA, Lin CP, et al. Optical coherence tomography. Science 1991;254:1178-81.

14 Huang D, Wang J, Lin CP, et al. Micron-resolution ranging of cornea and anterior chamber by optical reflectometry. Lasers Surg Med 1991;11:419-25.

15 Hee MR, Izatt JA, Swanson EA, et al. Optical coherence tomography of the human retina. Arch Ophthalmol 1995;113:325-32.

16 Hee MR, Izatt JA, Swanson EA, et al. Optical coherence tomography of the human retina. Arch Ophthalmol 1995;113:325-32.

17 Puliafito CA, Hee MR, Lin CP, et al. Imaging of macular diseases with optical coherence tomography. Ophthalmology 1995;102:217-29

18 Schuman JS, Hee MR, Puliafito CA, et al. Quantification of nerve fiber layer thickness in normal and glaucomatous eyes using optical coherence tomography. A pilot study. Arch Ophthalmol 1995;113:586-96.

19 Yannuzzi LA, Slakter JS, Sorenson JA, et al. Digital indocyanine green videoangiography and choroidal neovascularization. Retina 1992;12:191-223.

20 Yannuzzi LA, Hope-Ross M, Slakter JS. Analysis of vascularized pigment epithelial detachments using indocyanine green videoangiography. Retina 1994;14:99-113.

21 Hee MR, Baumal CR, Puliafito CA, et al. Optical coherence tomography of age-related macular degeneration and choroidal neovascularization. Ophthalmology 1996;103: 1260-70.

22 Puliafito CA, Hee MR, Schuman JS, et al. Optical coherence tomography of ocular diseases. Thorofare, NJ: Slack Inc, 1996:220-39.

23 Grossniklaus HE, Hutchinson AK, Capone A, et al. Clinicopathologic features of surgically excised choroidal neovascular membranes. Ophthalmology 1994;101:1099111.

24 Mietz H, Merrill PT, Lambert HM, et al. Combined subretinal and sub-retinal pigment epithelium neovascular membranes in age-related macular degeneration: a clinicopathologic study of six cases. Ophthalmic Surg Lasers 1997; 28:645-52. 